\title{
Papillary adenocarcinoma of the renal pelvis with renal calculus: A rare case report
}

\author{
JIANLONG LI ${ }^{1}$, QING $\mathrm{LI}^{2}$ and YI YU ${ }^{1}$ \\ Departments of ${ }^{1}$ Urology and ${ }^{2}$ Pathology, The Second Affiliated Hospital of Nanchang University, \\ Nanchang, Jiangxi 330046, P.R. China
}

Received October 30, 2015; Accepted January 20, 2016

DOI: $10.3892 / \mathrm{mco} .2016 .783$

\begin{abstract}
Papillary adenocarcinoma of the renal pelvis is a rare clinicopathology of a kidney tumor with renal calculus. In the present case report, percutaneous renal biopsy, nephroscope lithotripsy and radical nephroureterectomy within a papillary adenocarcinoma of the renal pelvis accompanied with renal calculus was performed on a 65 -year-old patient, also including a report on the patient's data and a literature review. The histopathological features confirmed the diagnosis of papillary adenocarcinoma of the renal pelvis. Tumors of the renal pelvis are uncommon features of urothelial carcinoma, and papillary adenocarcinoma of the renal pelvis is a very unusual entity. The present case report describes papillary adenocarcinoma of the renal pelvis with renal calculus, which has rarely been previously reported.
\end{abstract}

\section{Introduction}

Adenocarcinoma of the renal pelvis is a rare malignant type of renal tumor. Additionally, papillary adenocarcinoma of the renal pelvis with renal calculus is rarely reported in the literature. In the current study, a rare case report of papillary adenocarcinoma of the renal pelvis with renal calculus is presented, and the underlying clinical and pathological factors that may have resulted in the misdiagnosis are investigated. The aim of the present case study report was to focus the attention of clinicians on this type of cancer by providing our preliminary clinical experience in order to improve its diagnosis and treatment.

\section{Case report}

All procedures in the present case report were performed in accordance with the ethical standards of the institutional review board and ethics committee of the Second Affiliated

Correspondence to: $\mathrm{Dr} \mathrm{Yi} \mathrm{Yu}$, Department of Urology, The Second Affiliated Hospital of Nanchang University, No. 1 Minde Road, East Lake Area, Nanchang, Jiangxi 330046, P.R. China

E-mail: yuyiapple@126.com

Key words: papillary adenocarcinoma, renal pelvis, renal calculus
Hospital of Nanchang University, Nanchang, China, and with the 1964 Helsinki declaration. Written consent for using the samples for research purposes was obtained from the patient following surgery, and the ethics committee approved the consent procedure.

A 65-year-old woman presented with 20 years' history of renal calculus on the right side. The patient complained of pain, particularly over a one month period. Additionally, the patient had experienced a fever, with a temperature recorded as high as $38.5^{\circ} \mathrm{C}$ for a week. The patient did not present with observable symptoms, such as hematuria, urgency and urinary pain. The patient was prescribed a course of penicillin as an anti-inflammatory treatment in the local community hospital, although the symptoms showed no indications of any improvement as a consequence of the treatment.

The patient was referred to our urology facility, and was thoroughly examined, including undergoing abdominal computerized tomography (CT) using a Second Generation Dual-Source CT Scanner (SOMATOM Definition Flash; Siemens Healthcare AG, Munich, Germany), which revealed that the right kidney volume was increased, with renal cortical thinning. The right renal pelvis calyces were in a state of expansion and effusion. Several nodular density shadows were detected on the right of the calyces, with a clear border. The largest one measured $2.6 \times 2.5 \mathrm{~cm}$ (Fig. 1). The laboratory examinations revealed that the patient had urinalysis, suggesting the presence of a severe infection in the urinary tract, as did the blood cell analysis. In order to block the development of septic shock, an emergency percutaneous nephrostomy was performed. The stench of milky white pus was observed in the drainage tube. A culture of Escherichia coli bacteria was taken from the pus, which proved to be sensitive to piperacillin treatment ( $2.5 \mathrm{mg}$, two times/day). After 15 days' treatment of drainage and sensitive antibiotics, the patient was offered nephroscope lithotripsy through the former incision. However, a cauliflower-like neoplasm was identified on the mucosa of the right mid-lower group calyces. On the basis of the histopathological biopsy report, a diagnosis of tubular papillary adenocarcinoma was made. Hematoxylin and eosin (H\&E) staining revealed features of the papillary adenocarcinoma cells, including their adenoid arrangement, cellular pleomorphism and invasive growth. Further coronal CT angiography demonstrated a mass in the right renal pelvis (Fig. 2). After general anesthesia, the patient subsequently received radical 


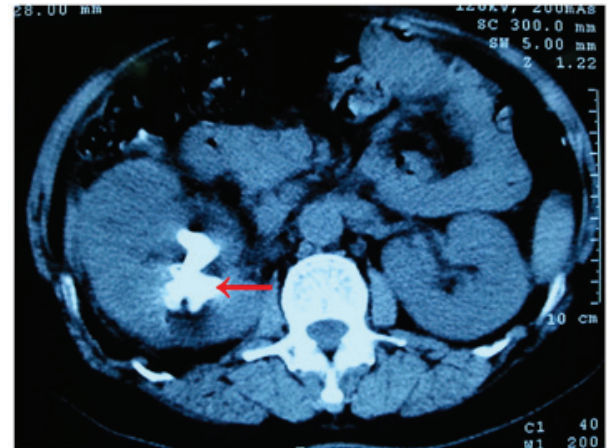

Figure 1. Axial abdominal computerized tomography, revealing the extensive renal calculus on the right-hand side (marked by the red arrow).

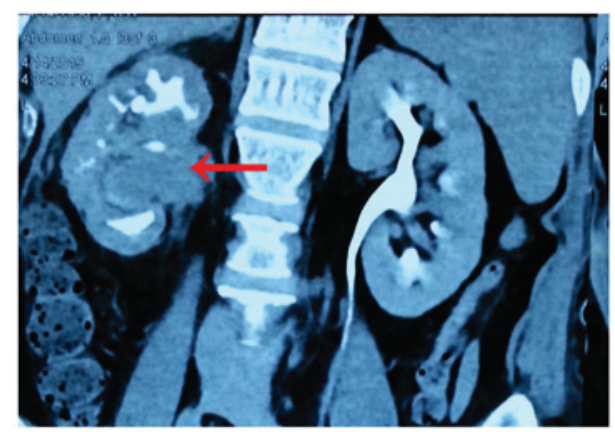

Figure 2. Coronal computerized tomographic angiography, revealing the presence of a mass in the right renal pelvis (marked by the red arrow).

nephroureterectomy. The gross specimen of the removed mass is shown in Fig. 3.

For the immunohistochemical examination, paraffin-embedded specimens were cut into $5 \mu \mathrm{m}$ sections and mounted onto poly-L-lysine-coated slides. The sections were deparaffinized, rehydrated, and subsequently boiled for $10 \mathrm{~min}$ in $10 \mu \mathrm{mol} / \mathrm{l}$ citrate buffer solution ( $\mathrm{pH}$ 6.0) using a microwave oven. Endogenous peroxidase was blocked with $0.3 \%$ hydrogen peroxide for $30 \mathrm{~min}$, and non-specific staining was blocked by treating the slides with $1 \%$ fish-skin gelatin for $30 \mathrm{~min}$ at room temperature. Subsequently, slides were incubated overnight with primary antibodies against 34E 312 , CAM5.2 and cytokeratin 19 (1:100; Abcam, Cambridge, UK). After washing with PBS, the slides were incubated with prediluted secondary antibody (Abcam), followed by further incubation with diaminobenzidine. Finally, the sections were counter-stained with H\&E stain and mounted. The immunohistochemical examination, following the radical nephroureterectomy, revealed the tumor to be a rare papillary adenocarcinoma. The patient returned to normal following the surgery, and made an uneventful recovery. The patient was normal at the 5-month follow-up stage.

\section{Discussion}

Tumors of the renal pelvis are uncommon in clinicopathology, and constitute $>5 \%$ of the malignancies arising from the renal mass. Furthermore, adenocarcinoma accounts for an extremely small percentage of neoplasms arising within the renal pelvis, with a relative frequency of only $1 \%$ (1).

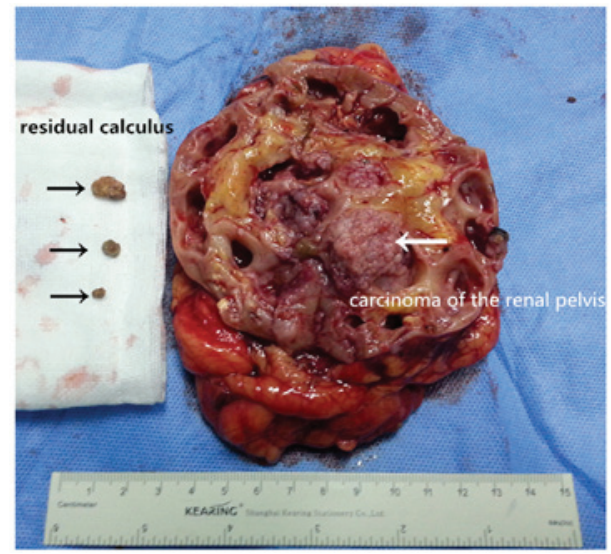

Figure 3. Photograph showing the gross appearance of the carcinoma (indicated by the white arrow) with residual calculus (shown by the black arrow) replacing the kidney.

Adenocarcinomas of the renal pelvis may be subdivided into three major histological types: Tubulovillous (71.5\%), mucinous $(21.5 \%)$ and papillary non-intestinal $(7 \%)$. Mucinous adenocarcinoma of the renal pelvis was first described in 1960 by Hasebe et al (2). They are occasionally considered to arise in the foci of intestinal metaplasia (2). According to the statistics, a total of 51 cases were reported of adenocarcinoma of the renal pelvis in the world by 1993, and reports of adenocarcinoma have been predominantly recorded as being of the mucinous type ever since $(3,4)$. However, papillary adenocarcinoma of the renal pelvis was described in Chinese journals, in 1987 by Fang (5) and in 1997 by Kong et al (6). Other reports of papillary adenocarcinoma do exist in other language journals, although these are rarer in occurrence. The finding, in 2005, that papillary adenocarcinoma of the renal pelvis and ureter produce carcinoembryonic antigen, carbohydrate antigen 19-9 and carbohydrate antigen 125 in metastatic adenocarcinoma was reported by Onishi et al (7). However, in the present case study, the serum levels of the above tumor biomarkers were normal, and none of the evidence from the imaging suggested the presence of gastrointestinal tumors in the patient. Immunohistochemical examination of the cytokeratin 34E $\beta 12(+)$, the anticytokeratin CAM5.2(+) and cytokeratin 19(+) demonstrated that the tumor originated in the glandular epithelium, and not in the loops of Henle. Therefore, it was hypothesized that the patient in the present case study only had primary papillary adenocarcinoma of the renal pelvis, which may be associated with renal calculus and chronic infection. This is in agreement with another study, which elucidated that adenocarcinoma of the renal pelvis is associated with lithiasis (7). Therefore, for future reference, this clinical case study has indicated that, firstly, a careful search for a papillary adenocarcinoma of the renal pelvis should be excluded from metastasis of the digestive tract. Subsequently, the possibility of long-term disease associated with an infection of the kidney calculus should be considered by screening the mucous membrane carcinoma of the renal pelvis. Finally, a consensus on standardized surgery, postoperative chemotherapy or radiotherapy of papillary adenocarcinoma in the renal pelvis should be reached by global urology researchers for the treatment of this disease. 


\section{Acknowledgements}

We would like to thank Professor Zimin Shi and Hongwei Huang at the Urology Department of the Second Affiliated Hospital of Nanchang University, and Professor Liqing Wu at the Pathology Department of the Second Affiliated Hospital of Nanchang University, for their constant encouragement with this study and for offering technology guidance.

\section{References}

1. Kaur G, Naik VR and Rahman MN: Mucinous adenocarcinoma of the renal pelvis associated with lithiasis and chronic gout. Singapore Med J 45: 125-126, 2004
2. Hasebe M, Serizawa S and Chino S: On a case of papillary cystadenocarcinoma following malignant degeneration of a papillary adenoma in the kidney pelvis. Yokohama Med Bull 11: 491-500, 1960.

3. Fareghi M, Mohammadi A and Madaen K: Primary mucinous cystadenocarcinoma of renal pelvis: A case report. Cases J 2: 9395, 2009

4. Xambre L, Cerqueira M, Cardoso A, Correia T, Macedo Dias A, Carreira F and Galán T: Primary mucinous adenocarcinoma of the renal pelvis-adicional case report. Actas Urol Esp 33: 200-204, 2009 (In Spanish).

5. Fang G: Tow case of papillary adenocarcinoma of renal pelvis with renal calculus. Guangxi Medicine 9: 216, 1987 (In Chinese).

6. Kong X, Xia T, Pan B, Xue Z, Guo Y and Gu F: A case of papillary adenocarcinoma of renal pelvis. Chin J Pathol 26: 127, 1997 (In Chinese).

7. Onishi T, Franco OE, Shibahara T, Arima K and Sugimura Y: Papillary adenocarcinoma of the renal pelvis and ureter producing carcinoembryonic antigen, carbohydrate antigen 19-9 and carbohydrate antigen 125. Int J Urol 12: 214-216, 2005. 